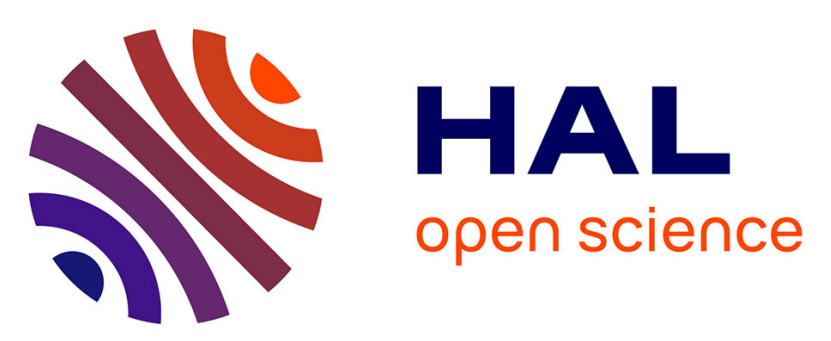

\title{
A real time affinity biosensor on an insulated polymer using electric impedance spectroscopy in dielectric microchips.
}

Mohammed Kechadi, Bruno Sotta, Lila Chaal, Bernard Tribollet, Jean Gamby

\section{- To cite this version:}

Mohammed Kechadi, Bruno Sotta, Lila Chaal, Bernard Tribollet, Jean Gamby. A real time affinity biosensor on an insulated polymer using electric impedance spectroscopy in dielectric microchips.. Analyst, 2014, 139 (12), pp.3115-3121. 10.1039/c4an00212a . hal-01019204

\section{HAL Id: hal-01019204 https: / hal.sorbonne-universite.fr/hal-01019204}

Submitted on 27 Nov 2014

HAL is a multi-disciplinary open access archive for the deposit and dissemination of scientific research documents, whether they are published or not. The documents may come from teaching and research institutions in France or abroad, or from public or private research centers.
L'archive ouverte pluridisciplinaire HAL, est destinée au dépôt et à la diffusion de documents scientifiques de niveau recherche, publiés ou non, émanant des établissements d'enseignement et de recherche français ou étrangers, des laboratoires publics ou privés. 


\title{
Real time affinity biosensor onto insulated polymer using electric impedance spectroscopy in dielectric microchip
}

\author{
Mohammed Kechadi, ${ }^{\text {a,b,c }}$ Bruno Sotta, ${ }^{\mathrm{d}}$ Lila Chaal, ${ }^{\mathrm{c}}$ Bernard Tribollet, ${ }^{\mathrm{a}, \mathrm{b}}$ and Jean Gamby ${ }^{\mathrm{a}, \mathrm{b}, *}$
}

\author{
This paper presents development real time monitoring of binding events onto flexible plastic in \\ microchips. Two planar carbon microelectrodes are integrated into insulated polyethylene \\ terephthalate microchip without direct electrical contact with the solution in microchannel. It has been \\ possible to probe the electric impedance changes through the interface constituted by the \\ microelectrode/ PET microchannel/solution when a biomolecular interaction takes place onto the \\ ${ }_{10}$ polymer surface. This new transduction for biosensing was demonstrated for the molecular recognition \\ of BSA immobilized on the polymer microchannel surface using the corresponding rabbit anti-BSA \\ antibodies as an analyte in the flow microchannel at the nanomolar range concentration. The \\ equilibrium association constant was determined for the affinity reaction between both ligands and \\ was obtained equal to $5 \times 10^{7} M^{-1}$. The promising results obtained with this new device make it a \\ 15 competitive biosensor.
}

\section{Introduction}

The last decades have seen the emergence of microfluidic devices with various tranducers for affinity biosensor development. The 20 most used tranducers are electrochemical ${ }^{[1],[2]}$, electroacoustic ${ }^{[3],[4]}$, or optical ${ }^{[5],[6]}$ for detecting interactions between ligands. Surface plasmon resonance (SPR) is one of the most used optical biosensors for the measurement of the refractive index changes ${ }^{[7],[8]}$. As illustrated in Figure 1A with Biacore SPR instrument 25 scheme, the ligand (antigen, receptor...) is immobilised in the hydrogel, while the analyte (antibody, messenger...) flows in the channel and diffuses in the gel where the ligand is bound. The metal/ dielectric interface constituted to glass slide coated with hydrogel and gold is excited by a beam of monochromatic light, 30 in conditions of total reflection leading to the resonance. The light beam probes the hydrogel and detects changes in the local refractive index due to the accumulation of analyte mass. The depth of the evanescent wave is $100 \mathrm{~nm}$ towards the hydrogel, i.e. the thickness of the hydrogel itself.

35 In the last decades, several groups have focused on electrical signal transduction in microchip by using direct or indirect electrode configuration to measure the conductivity of the streaming electrolyte in microchannel. An original technique has been the development of measurements through capacitively 40 coupled contactless conductivity detection $\left(C^{4} D\right)$ through two electrodes with a distance separation constituting the detector cell $[9],[10],[11]$. However, the high applied ac voltage $0.5-10 \mathrm{~V}$ amplitudes are much larger than conventionally used values, $5 \sim$ $100 \mathrm{mV}$. Such large amplitude generates a non-linear relationship 45 between voltage and current which cause distortion of impedance results. It is for this reason that this transduction can be viewed as pseudoimpedance or transimpedance technique.

In recent years, laser photoablation technique was used to defined a well-controlled microelectrodes position in polymer microchip. s0 The feeble gap between the microelectrodes as well as with the thin layer of dielectric material that coated the microelectrode allowed to avoid the linearity problem ${ }^{[12]}$. As mentioned for SPR, the principle of the developed electric impedance in dielectric microchip is also based on the metal/ dielectric interface 55 excitation by applying a modulated voltage of one hundred of millivolts in frequency range through the dielectric layer. The output impedance through the device can thus be related to the interfacial impedance of the modified dielectric layer sandwiched between the microelectrodes and the flow microchannel (see ${ }_{60}$ Figure 1B). The crucial advantage of electric impedance spectroscopy on insulated polymer sandwiched between two microelectrodes is the absence of direct contact between the microelectrodes and the flow microchannel ${ }^{[12],[13]}$. As a consequence the polymer microchannel layer sandwiched ${ }_{65}$ between the two planar microelectrodes can be considered as a dielectric component ${ }^{[14]}$ whose physical properties may change in response to an external electrical excitation. Based on this principle, in a previous paper, a flow sensor was developed to record at high fixed frequency the chemical conversion of 70 enzymatic substrate like aminophenyl phosphate in PET microchannel through the channel resistivity ${ }^{[15]}$. In this paper, we demonstrate for the first time the possibility to probe the interfacial electric impedance change when a biomolecular interaction takes place between a ligand in solution (analyte) with 75 a ligand attached onto the polymer microchannel. This new transduction for biosensing was demonstrated for the detection and quantification of bovine serum albumin (BSA) immobilized on the polymer microchannel surface using the corresponding rabbit anti-BSA antibodies (Ab-BSA) as an analyte, which is 80 loaded in the flow microchannel at nanomolar range concentration. Experimental studies are based on the real time monitoring of the measured impedance variation at a fixed frequency $(400 \mathrm{~Hz})$ by applying a small $100 \mathrm{mV}$ alternating potential excitation through two microband electrodes, during the 85 association/ dissociation rate between Ab-BSA loaded in flow microchannel and BSA immobilized on PET. 


\section{Materials and methods}

\subsection{Chemicals}

$10 \mathrm{mM}$ of Tris(hydroxymethyl)aminomethane (from Sigma Aldrich) was obtained after dilution in $10 \mathrm{mM} \mathrm{HCl}$ and $10 \mathrm{mM}$ ${ }_{5} \mathrm{NaCl}$. The checked $\mathrm{pH}$ was at 9 and was used as the buffer solution. 1mM NaOH (from Sigma Aldrich) was used as washing buffer in a regenerating step. Bovine serum albumin (96\%) was purchased from VWR International. The rabbit anti-bovine serum albumin antibodies (Ab-BSA) was received from Sigma-Aldrich.

10 The antibody solutions were also diluted in the buffer solution from a $10^{-5} \mathrm{M}$ Ab-BSA stock solution in $50 \%$ glycerol. Tween 20 solution was used with the ratio: Tween 20 /buffer solution $=0.2$ $\mathrm{ml} . \mathrm{L}^{-1}[16]$.

\subsection{Microchannel Networks}

15 Photoablation procedure for the microchannel networks has been previously described ${ }^{[17],[12]}$. In brief, the microchip used in this work was realised by photoablation process which leads to the ablation of the material in contact with UV laser source. Before the photoablation step, the PET film thickness was measured at $20100 \mu \mathrm{m}$. On the one side of the PET sheet two parallel microchannels (which will be transformed into microelectrodes) were drilled. The two microchannels depth into PET film was measured under microscope at $50 \mu \mathrm{m}$. On the other side of the PET sheet, a perpendicular microchannel for fluidic was drilled

25 having a trapezoidal cross-section shape with a depth of $45 \mu \mathrm{m}$, a width of $100 \mu \mathrm{m}$ and a length of $1.4 \mathrm{~cm}$ (See Figures 1B and C). Thus, a $5 \mu \mathrm{m}$ thick PET layer separated the microchannel from the bottom of the two microelectrodes (See Figure 1B). The two parallels microchannels were transformed into microelectrodes by 30 incorporation of a graphite carbon ink then dried afterward at $50^{\circ} \mathrm{C}$. Finally the photo-ablated PET film was laminated using a $35 \mu \mathrm{m}$-PE/PET layer. As shown in Figures $1 \mathrm{~B}$ and $\mathrm{C}$, the distance separation between both microelectrodes is $120 \mu \mathrm{m}$ edge to edge (200 $\mu \mathrm{m}$ center to center) and the detection surface area per 35 microelectrode is $80 \mu \mathrm{m} \times 100 \mu \mathrm{m}$. Study was carried out by the electric impedance measurement in alternative mode (ac) through the two microelectrodes at frequency range from $1 \mathrm{MHz}$ to $1 \mathrm{~Hz}$ with $0.1 \mathrm{~V}$ amplitude, in order to control the dielectrical behaviour of the overall microsystem and to extract the specific 40 microchannel response from the background response ${ }^{[18]}$.

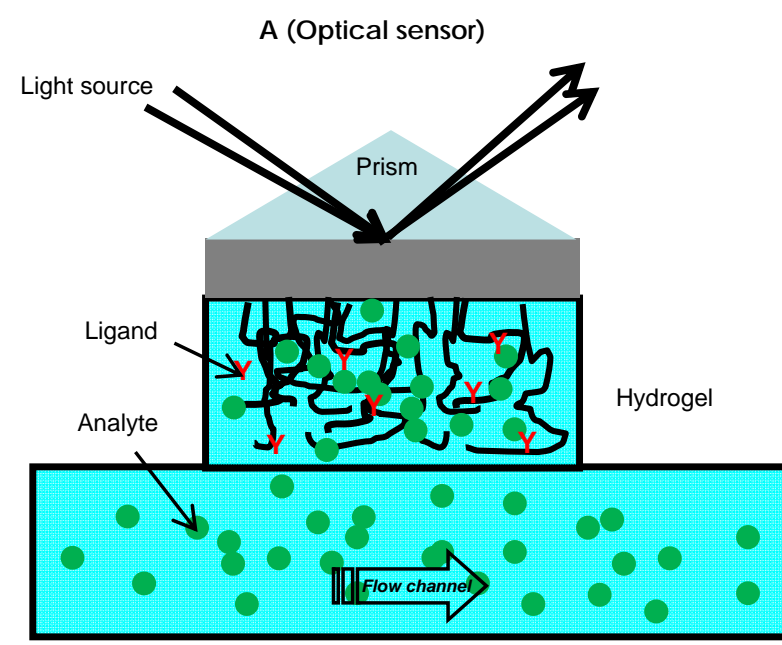

B (Electrical sensor)

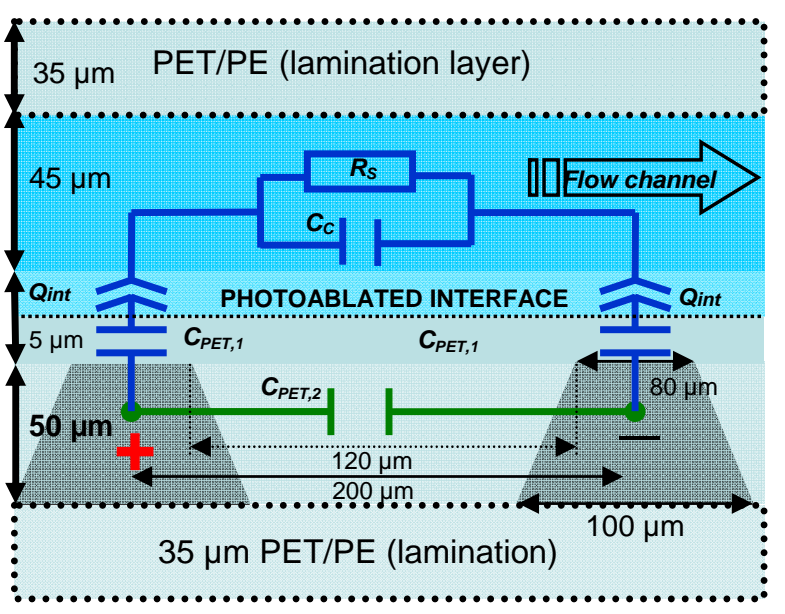

C (Detection zone zoomed)

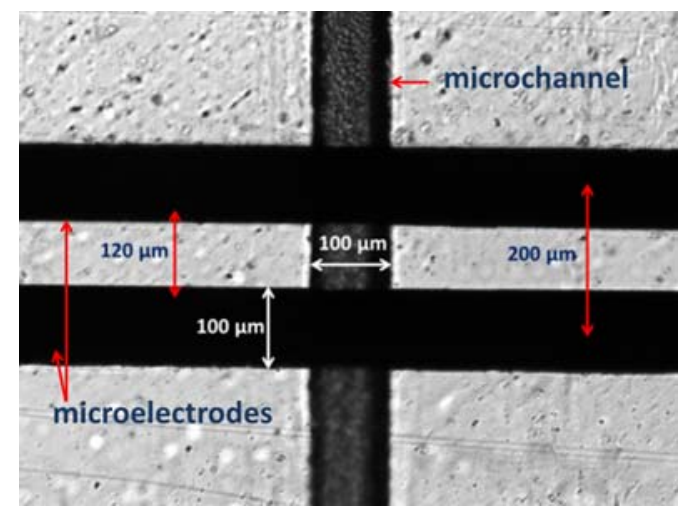

Figure 1. A. Schematic representation of the BIACORE optical biosensor. The hydrogel is $100 \mathrm{~nm}$ high approximately; the flow channel for a 50 standard instrument is $24 \mathrm{~mm}$ long, $50 \mu \mathrm{m}$ high and $500 \mu \mathrm{m}$ width. The ligand is immobilised in the hydrogel, the analyte flows in the channel and diffuses in the gel where it binds to the ligand. B. Cross section of the dielectric interface microelectrode/ PET/ microchannel and the corresponding electrical equivalent circuit: $C_{\mathrm{PET}, 2}$ for the $120 \mu \mathrm{m}$-PET

55 layer impedance (distance separation), $C_{\mathrm{PET}, 1}$ for the $5 \mu \mathrm{m}$-PET layer impedance (non-contact layer thickness), the element CPE, Qint, for the interfacial impedance (photoablated surface), and the parallel association of the solution resistance, $R_{\mathrm{S}}$, and the cell capacitance, $C_{\mathrm{C}}$. Please note 
that for a more detailed view of the interface the dimensions are not to scale. C. The zoomed detection is taken under an inverted microscope.

\subsection{Instrumentation}

5 Impedance measurements are performed using Dielectric interface (Solartron, DI 1260) gain phase impedance analyzer coupled with a frequency response analyzer (Solartron, FRA 1255). Dielectric interface is characterised by the large frequency range, from $1 \mathrm{mHz}$ to $20 \mathrm{MHz}$. Software Smart (Solartron 10 Analytical) was used as the computer control of all experimental set-up. Dielectric interface is usually used for high impedance measurement on high insulated material that is the case with polyethylene terephthalate. Indeed, current measurement range of the system varies from $6 \mathrm{~mA}$ to $100 \mathrm{fA}$, thus enabling the 15 measurement of very high impedance ( $100 \Omega$ to $100 \mathrm{~T} \Omega)$. In this study, impedance measurement is making at low fixed-frequency (here $400 \mathrm{~Hz}$ ) which was appropriate for sensing the interfacial impedance through the dielectric microchip device connected to a dielectric interface apparatus.

\subsection{Experimental procedure for electric impedance measurements in dielectric microchip}

The microchip characteristics with an insulated PET layer sandwiched between two galvanic isolated microelectrodes can be assimilated to a dielectric interface with, on the one part of the ${ }_{25} 5 \mu \mathrm{m}$-PET thin polymer layer, electronic charges on microelectrodes and, on the other part, ionic charges in the microchannel considering impedances for each part of the microchip (see Figure 1B).

The global impedance $Z_{\mathrm{G}}(\omega)$ between the two microelectrodes 30 with a filled microchannel depends on the different parts which constitute the microchip. In this configuration, $Z_{G}(\omega)$ can be expressed as the combination between $Z_{1}(\omega)$ (blue line in Figure $1 \mathrm{~B})$ and $Z_{2}(\omega)$ (green line in Figure $\left.1 \mathrm{~B}\right)^{[18]}$, as expressed as follows,

${ }_{35} Z_{G}(\omega)^{-1}=Z_{1}(\omega)^{-1}+Z_{2}(\omega)^{-1}$

Firstly, acquisition and interpretation of electric impedance are performed with an empty microchannel through a $100 \mathrm{mV}$ voltage excitation applied between the two carbon microelectrodes embedded in PET and separated to $200 \mu \mathrm{m}$ 40 center to center $(120 \mu \mathrm{m}$ edge to edge, Fig. 1B). The $120 \mu \mathrm{m}$ PET impedance layer, $Z_{2}(\omega)$, which separated two electrodes behaves as a capacitor (see equation 2 ) and will be eliminated from the global impedance, $Z_{\mathrm{G}}(\omega)$, in further experiments when microchannel will be filled. The capacitance written in equation 2 45 was obtained at $C_{\mathrm{PET}, 2}=8 \times 10^{-13} \mathrm{~F}$.

$$
Z_{2}(\omega)=\frac{1}{j \omega C_{P E T, 120 \mu m}}
$$

where $\omega$ is the angular frequency $\left(\mathrm{rad} \mathrm{s}^{-1}\right)$, defined by $\omega=2 \pi f$, where $f$ is the frequency.

The target application is the real-time monitoring of the electric 50 impedance module variation $Z_{1}(\omega)$ at low frequencies. The latter impedance as appears in Figure 1B corresponds to a serial combination of three impedances as written below:

$$
Z_{1}(\omega)=2 Z_{1, P E T}(\omega)+2 Z_{\text {int }}(\omega)+Z_{\text {microchannel }}(\omega)
$$

The first term in equation 3 is the $5 \mu \mathrm{m}$-PET layer impedance 55 from the top of one electrode to the channel bottom that behaves also as $C_{\mathrm{PET}, 120 \mu \mathrm{m}}$ (see equation 2). The value of $C_{\mathrm{PET}, 5 \mu \mathrm{m}}$ equals $14 \times 10^{-11} \mathrm{~F}$, is fixed and will be suppressed during the normalized procedure. The second term displayed in equation 3 is the microchannel solution impedance, $Z_{\mathrm{S}}$, that corresponds to a ${ }_{60}$ parallel association between the microchannel solution resistivity, $R_{\mathrm{S}}$, and the cell capacitance, $C_{\mathrm{C}}$. The resistance $R_{\mathrm{S}}$ only depends on the buffer concentration that is high in comparison with the analyte concentration. The capacitance, $C_{\mathrm{C}}$, is defined as a geometric capacitor that only depends on the chip dimension. ${ }_{65}$ This latter was equal to $C_{\mathrm{C}}=3 \times 10^{-13} \mathrm{~F}$. The last term represents the interfacial impedance, $Z_{\text {int }}$, at the photoablated PET/ solution interface. As obtained in previous paper ${ }^{[19]}$, this latter behaves as a non ideal capacitance represented by a constant phase element (CPE) where $\alpha_{\text {int }}$ and $Q_{\text {int }}$ are the CPE exponent and the CPE 70 element (see equation 4 ), respectively.

$$
Z_{\text {int }}(\omega)=\frac{1}{(j \omega)^{\alpha_{\text {int }}} Q_{\text {int }}}
$$

To conclude, $Z_{\text {int }}$ is the only interesting parameter to monitor (see equation 3) which varies when a biomolecular interaction takes 75 place on the photoablated PET surface throughout the course of the experiment. As a consequence, the real time monitoring of $Z_{\text {int }}$ can be measured through the relative variation of $Z_{1}$. Indeed, this latter at low frequencies mainly reflects the contribution of the interfacial impedance as written in equation 5 .

${ }_{80} \Delta Z_{1}(\omega)=\Delta Z_{\text {int }}$

\subsection{Experimental procedure for adsorption monitoring}

In the first step, PET microchannel was coated under a laminar flow contained $10^{-7} \mathrm{M}$ BSA for $1200 \mathrm{~s}$ in order to saturate the 85 microchannel surface. The solutions were loaded into the microchannel using high precise micropump with a flow rate equal to $F_{v}=7.2 \mu \mathrm{L} \mathrm{m^{-1 }}$. Indeed this optimized BSA concentration was chosen because it corresponds to the experimental plateau for BSA adsorbed onto PET surface ${ }^{[20]}$. 90 Before loading the antibody, Tween 20 solution was also introduced over $1200 \mathrm{~s}$ to prevent the non specific adsorption of Ab-BSA in the molecular recognition step ${ }^{[16]}$. The molecular recognition step was the introduction of various Ab-BSA solution (from $10^{-8}$ to $10^{-7} \mathrm{M}$ ) in the same hydrodynamic conditions. The 95 last step consisted in a washing procedure with $10^{-3} \mathrm{M} \mathrm{NaOH}$ for PET surface regeneration [20],[21],[22]. This procedure allows the start baseline recovering when water and the buffer solution were introduced again for a new started run experiment.

\section{Results and discussion}

\subsection{Affinity reaction rate determination onto PET microchannel}

A typical variation of the interfacial impedance module when Ab- 
BSA solution is loaded on a prepared PET microchannel surface (BSA then Tween 20) is displayed in Figure 2. The initial value corresponds to the electric impedance module baseline obtained after BSA and Tween 20 samples were successively loaded in the 5 microchannel (named $Z_{\mathrm{BSA}}$ ). First, a control experiment was performed showing no significant changes on the measured interfacial impedance modulus when no rabbit anti-BSA antibodies are present in buffer solution (Figure 2, curve a). Secondly, the measured interfacial impedance modulus increases 10 in the presence of Ab-BSA diluted with the buffer solution while the affinity reaction with BSA takes place. Then, the interfacial impedance signal response reaches a plateau value corresponding to a stationary signal (named $Z_{\mathrm{Ab}-\mathrm{BSA}}$ ). A statistical study about the changes on interfacial impedance modulus in presence of Ab15 BSA is given in Table $\mathrm{S} 1$ (See the supplementary material).

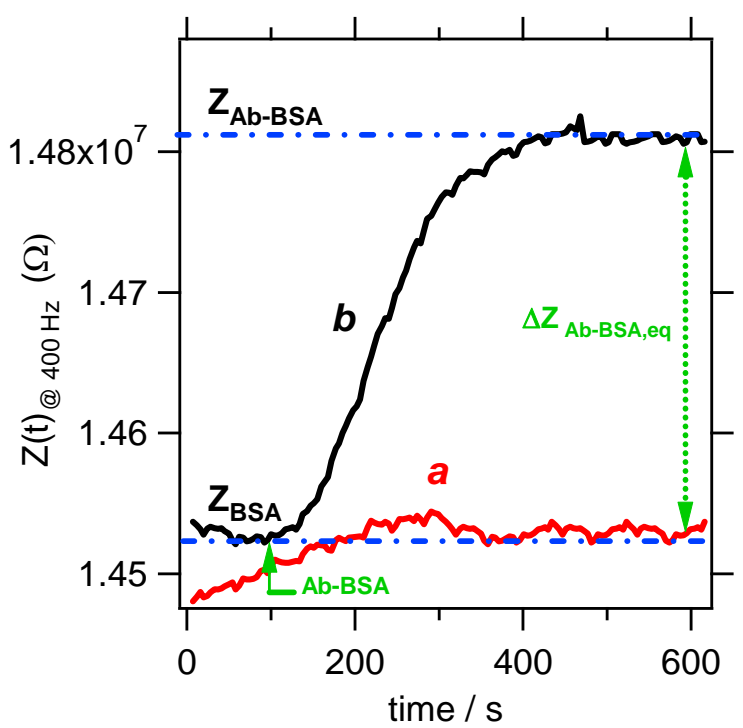

Figure 2. Sensorgrams showing the impedance module versus time Curve a: A control experiment when no rabbit anti-BSA antibodies are present in buffer solution. Curve b: The association step between Ab-BSA 20 as analyte and the adsorbed BSA on the microchannel sensor at a fixed frequency of $400 \mathrm{~Hz}$ and for a flow rate $7.2 \mu \mathrm{L} \cdot \mathrm{min}^{-1}$. Step 1: BSA and Tween 20 in buffer solution for microchannel coating. Step 2: Introduction of $4 \times 10^{-8} \mathrm{M}$ Ab-BSA diluted in the buffer solution.

The difference between the two stationary signals leads to an 25 equilibrium status named $\Delta Z_{\mathrm{Ab}-\mathrm{BSA} \text {,eq }}$ which is equal to $Z_{\mathrm{BSA}}-Z$ Ab-BSA. Plotting the equilibrium binding obtained from the difference values $\Delta Z_{\text {Ab-BSA,eq }}$ against various loaded Ab-BSA concentrations leads to an isotherm behaviour displayed in Figure $3 \mathrm{~A}$. Indeed, as the reaction binding progresses, the rate decreases 30 gradually until a state that corresponds to an equilibrium between association and dissociation rates. The maximum value obtained for $\Delta Z_{\mathrm{Ab}-\mathrm{BSA} \text {,eq }}$ is more quickly reached while Ab-BSA concentration is high. We obtained a maximum value of $\Delta Z_{\mathrm{Ab}}$ BSA,eq $\left(\right.$ named $\Delta Z_{\mathrm{Ab}-\mathrm{BSA} \text {,max }}$ ) from a Ab-BSA concentration equals 35 to $5 \times 10^{-8} \mathrm{M}$. This means that all the active sites of BSA molecules adsorbed onto PET microchannel are saturated after the biomolecular recognition with Ab-BSA.

In order to find the model parameters corresponding to the binding reaction between Ab-BSA with BSA determined by 40 impedance measurements, the relative equilibrium binding curve is plotted against Ab-BSA concentration after normalization of
$\Delta Z_{\mathrm{Ab}-\mathrm{BSA} \text {,eq }}$ values divided by $\Delta Z_{\mathrm{Ab}-\mathrm{BSA}, \max }$ (displayed in the rightaxis of Figure $3 \mathrm{~A}$ ). As described previously, for Ab-BSA concentrations lower than $5 \times 10^{-8} \mathrm{M}$, the equilibrium binding 45 varies linearly. The Langmuir equation model ${ }^{[23]}$ has been tested to fit the experimental data synthetized in Figure 3 without good correlation. However, as yet known for heterogeneous sites for adsorption, the adapted Langmuir model named LangmuirFreundlich model ${ }^{[20],[24],[25]}$ was more adequate for the fitting so procedure to determine the kinetics parameters.
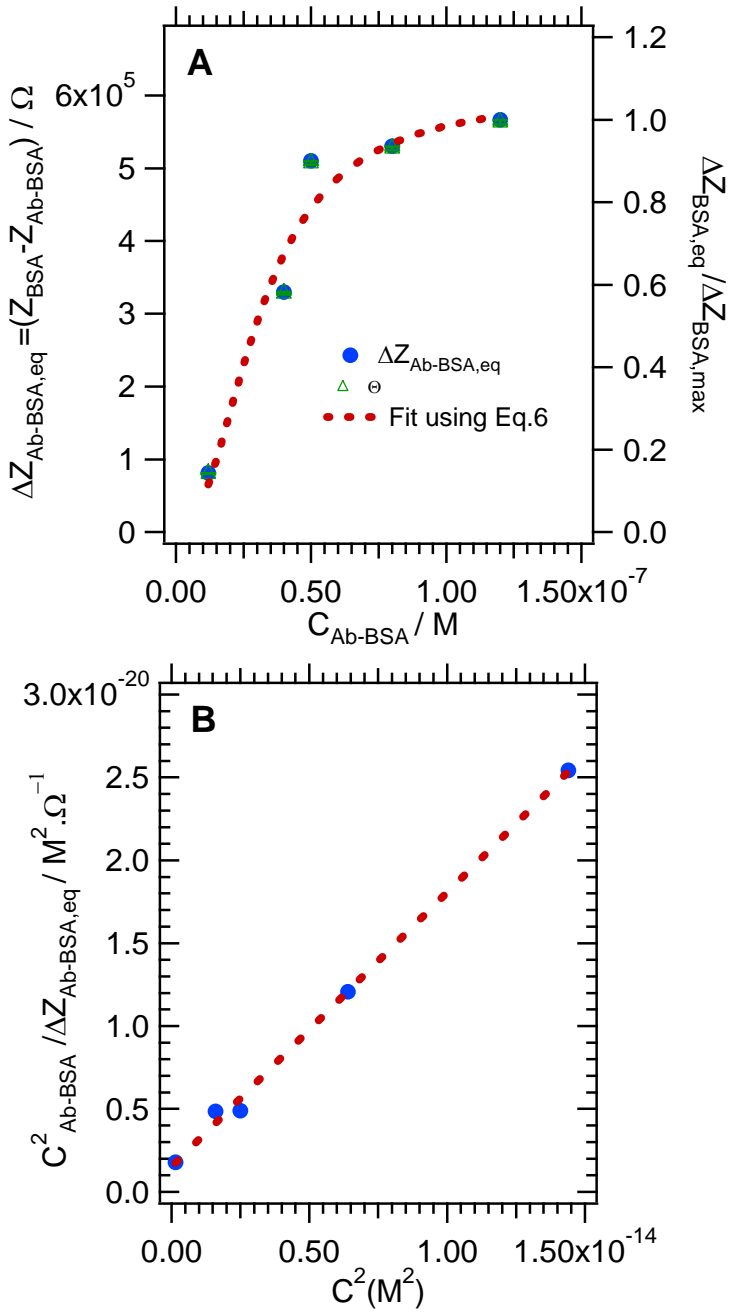

Figure 3. Calibration curve obtained for various Ab-BSA concentration loaded on the coated PET surface with BSA. (A) An association isotherm of Ab-BSA on the adsorbed BSA obtained from various experimental 55 sensorgrams from $12 \mathrm{nM}$ to $120 \mathrm{nM}$ concentration. (B). Linearization step of the association isotherm using a linear fit as indicated in equation 7.

Briefly, in the described experiment, the impedance normalized in Figure $3 \mathrm{~b}$ was assumed to be in linear correlation with the surface concentration of occupied sites. As a consequence, the ${ }_{60}$ Langmuir-Freundlich combined expression can be expressed as follows in equation 6 :

$$
\theta\left(C_{A b-B S A}^{m}\right)=\frac{\Delta Z_{A b-B S A, e q}}{\Delta Z_{A b-B S A, \max }}=\frac{K_{B S A / A b-B S A}^{a p p} C_{A b-B S A}^{m}}{1+\left(K_{B S A / a A b-B S A}^{a p p} C_{A b-B S A}^{m}\right)}
$$

where $\theta\left(\mathrm{C}^{m}{ }_{\mathrm{Ab}-\mathrm{BSA}}\right)$ can be viewed as the capacity of the 
microchannel to reach the maximum coverage, $m$ exponent represents the power term of Freundlich, and the apparent equilibrium constant, $K_{B S A / A b-B S A}^{a p p}$.

As traditionally admitted for the Langmuir equation ${ }^{[23]}$, a linear 5 expression of equation 6 is a useful test for the model validation as follows in equation 7 :

$$
\frac{C_{A b-B S A}^{m}}{\Delta Z_{A b-B S A, e q}}=\frac{1}{K_{B S A A / A b-B S A}^{a p p} \Delta Z_{A b-B S A, \max }}+\frac{C_{A b-B S A}^{m}}{\Delta Z_{A b-B S A, \max }}
$$

In order to obtain the traditional unit in $\mathrm{M}^{-1}$ for the equilibrium constant $K_{B S A / A b-B S A}$ the following relation must be taking into 10 account:

$$
K_{B S A / A b-B S A}^{a p p}=\frac{K_{B S A / A b-B S A}}{[A b-B S A]}
$$

As predicted, a better fit of experimental data is obtained with the Langmuir-Freundlich equation and the obtained parameters are equal to $K_{B S A /-A b-B S A}^{a p p}=5 \times 10^{15} \mathrm{M}^{-2}$ and $m=2$.

15 By replacing relation 8 in equations 6 and 7 , we recover the typical Langmuir equation model with a power term corresponding to the unit $(m=1)$. The linearization step of the association isotherm using a linear fit as indicated in equation 7 leads to a straight line with a correlation coefficient $\mathrm{R}^{2}$ equals to 20 0.996. Indeed, the curve fitting highlights a straight line characterized by a slope of $1.65 \times 10^{-6} \Omega^{-1}$ and an intercept of $1.51 \times 10^{-21} \mathrm{M}^{2} . \Omega^{-1}$. From these values the parameters of equation 6 were obtained equal to $m=2, \Delta Z_{A b-B S A, \max }=0.606 \mathrm{M} \Omega$ and $K_{B S A /-A b-B S A}^{a p p}=1.1 \times 10^{15} \mathrm{M}^{-2}$.

25

\section{2 kinetic model for affinity reaction onto polymer microchannel}

The interaction model for the binding reaction between Ab-BSA and BSA can be defined in the reaction scheme 1 below:

${ }^{30} B S A_{a d s}+(A b-B S A)_{\text {solution }} \underset{k_{o f f}}{\stackrel{k_{\text {on }}}{\Leftrightarrow}}\left(B S A_{a d s}-(A b-B S A)\right)_{\text {associated }}$

As demonstrated previously, results involve that the observed association rate, $v_{\text {obs }}$, between Ab-BSA and BSA can be expressed as a second order reaction proportional to the square of $\mathrm{Ab}$-BSA concentration, as follows:

$35 v_{o b s}=k_{o b s}[A b-B S A]^{2}$

with $v_{o b s}$ in $\mathrm{M} \mathrm{s}^{-1}$ and $k_{o b s}$ in $\mathrm{M}^{-1} \mathrm{~s}^{-1}$ represents the observed association kinetic rate constant.

Taking into account the concentration influence in the observed second order, the association kinetic rate constant can be 40 determined as follows:

$$
k_{\text {on }}=k_{\text {obs }}[A b-B S A]
$$

with $k_{o n}$ in $\mathrm{s}^{-1}$.

The dissociation rate of $\mathrm{BSA}_{\mathrm{ads}}-(\mathrm{Ab}-\mathrm{BSA})$ complex, $v_{\text {off }}$, is proportional with the $\mathrm{BSA}_{\mathrm{ads}}-(\mathrm{Ab}-\mathrm{BSA})$ concentration and it is ${ }_{45}$ considered as a zero order reaction since the amount of the adsorbed complex $\mathrm{BSA}_{\mathrm{ads}}-(\mathrm{Ab}-\mathrm{BSA})$ onto PET microchannel can be considered as a constant:

$v_{o f f}=k_{\text {off }}$

with $k_{\text {off }}$ in $\mathrm{M} \mathrm{s}^{-1}$ represents the dissociation kinetic rate constant so As a consequence the ratio $\left(k_{\mathrm{on}} / k_{\mathrm{off}}\right)$ is defined as the equilibrium constant, $K_{\mathrm{BSA} / \mathrm{Ab}-\mathrm{BSA}}$ which is defined in $\mathrm{M}^{-1}$.

The aim is now to determine by a full fitting procedure of the sensorgram impedance curve (see Figure 2), the kinetic parameters $k_{\text {on }}$ and $k_{\text {off }}$ leading to an estimation of the equilibrium ${ }_{55}$ constant of the complex $\mathrm{BSA}_{\mathrm{ads}}-(\mathrm{Ab}-\mathrm{BSA})$. For that, a theoretical expression of the impedance module $Z(t)$ at fixed frequencies with time can be written taking into account that the initial amount BSA adsorbed is related to impedance $Z_{\mathrm{BSA}}$ at the time $t=0$, as follows:

${ }^{60} Z(t)-Z_{B S A}=\Delta Z_{A b-B S A, \max }\left(\frac{k_{o n} C_{A b-B S A}}{k_{o n} C_{A b-B S A}+k_{o f f}}\right) \cdot\left[1-\exp \left(-\left(k_{o n} C_{A b-B S A}+k_{o f f}\right) t\right)\right]$

where $\mathrm{Z}(t)$ in $\Omega$ is the impedance module measured at fixed frequency $400 \mathrm{~Hz}$.

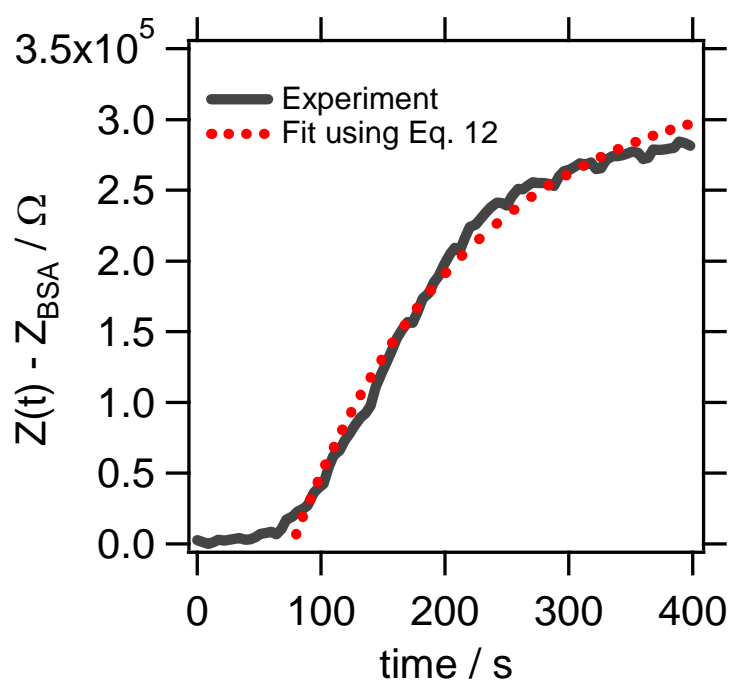

Figure 4. Experimental and fitted sensorgram of interfacial impedance modulus for $4 \times 10^{-8} \mathrm{M}$ of Ab-BSA loaded in the flow microchannel sensor showing the association reaction between Ab-BSA and the adsorbed BSA at a fixed frequency of $400 \mathrm{~Hz}$ and for a flow rate of $7.2 \mu \mathrm{L} \mathrm{min}{ }^{-1}$. The 70 curve fit was performed with equation 12 leading $k_{o n}, k_{o f f}$ equal to $8.56 \times 10^{4} \mathrm{~s}^{-1}$ and $3 \times 10^{-3} \mathrm{M} \mathrm{s}^{-1}$, respectively.

The result of the fitting procedure using equation 12 is illustrated in figure 4 with a working Ab-BSA concentration taken at $4 \times 10^{-8}$ $\mathrm{M}^{-1}$. The obtained values of $k_{\text {on }}$ and $k_{\text {off }}$ are $8.56 \times 10^{4} \mathrm{~s}^{-1}$ and $3 \times 10^{-}$ ${ }_{75}{ }^{3} \mathrm{M} \mathrm{s}^{-1}$ respectively. The same procedure was applied for various Ab-BSA concentrations and values of $k_{\text {on }}$ and $k_{\text {off }}$ have been determined for each curve (see Table S2 in the supplementary material). All the kinetic data obtained are synthetized in Figure $5 \mathrm{~A}$. It can be noticed that the values of $k_{\text {on }}$ and $k_{\text {off }}$ are slightly 80 dependent on the Ab-BSA concentration while the ratio, $k_{o n} / k_{\text {off }}$, is as expected, less dependent with Ab-BSA concentration, as appear in Figure 5B. This latter underlines that an average value for $K_{B S A / A b-B S A}$ can be estimated at $(5.15 \pm 1.45) \times 10^{7} \mathrm{M}^{-1}$, that is in 
a good agreement with the literature ${ }^{[26]}$. It is observed that the average value is very close to the one obtained with the higher Ab-BSA concentration i. e. $1.2 \times 10^{7} \mathrm{M}^{1}$.
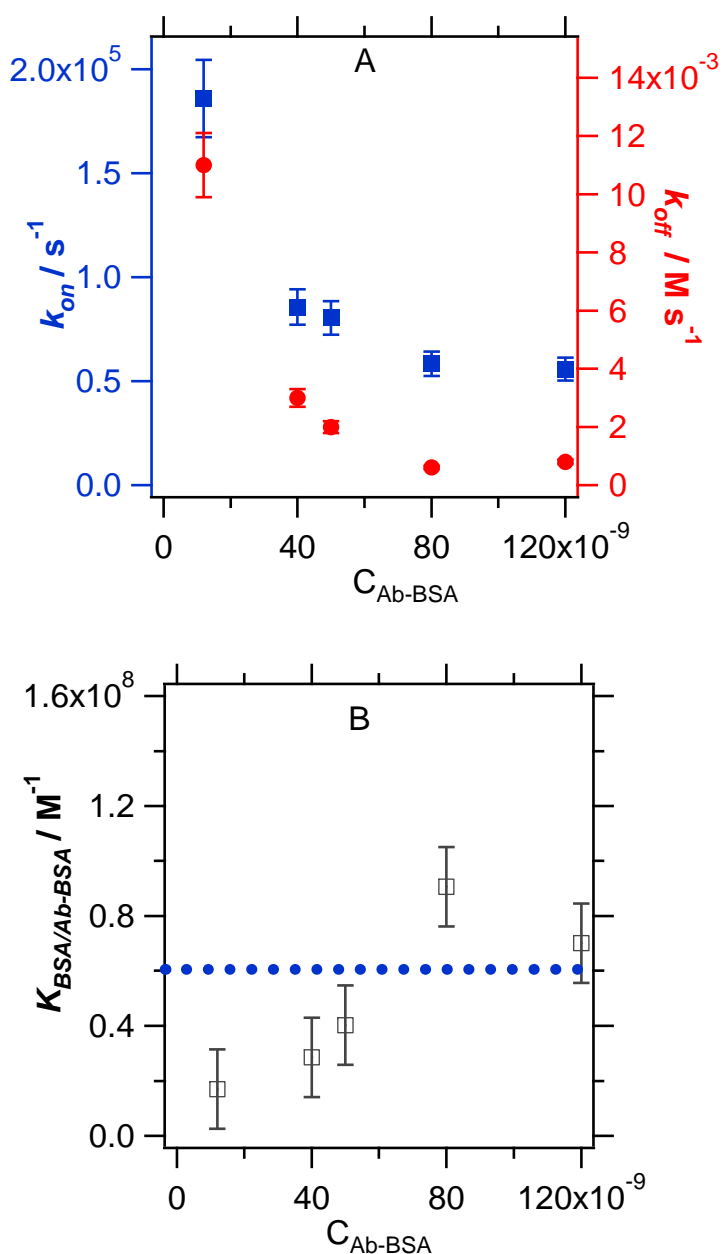

${ }_{5}$ Figure 5. Kinetic parameters obtained from the fitted sensorgrams of interfacial impedance modulus with several Ab-BSA (from $1.2 \times 10^{-8}$ to $1.2 \times 10^{-7}$ ) loaded in the flow microchannel sensor coated with BSA. A. The values obtained were from fitting procedure using equation 12 leading $k_{o n}$ and $k_{\text {off }}$ parameters which appear on left-axis and right-axis, 10 respectively. B. The equilibrium constant corresponding to each $k_{o n} / k_{o f f}$ ratio is indicated for each Ab-BSA concentration (See Table 2 in the supplementary material).

\section{Concluding Remarks}

15 Electric impedance spectroscopy in microchip was applied for the first time to monitor the biomolecular recognition of two ligands on insulated polymer designed as a microchip. As shown, the presence of BSA molecules immobilized onto the microchannel detection zone increases the interfacial impedance module

20 leading to a sensitive surface for biosensing in the nanomolar range concentration. The high sensitivity is found because this transduction takes advantage of the dielectric properties of the flow microchannel embedded in PET with two planar microband electrodes without contact with the solution, thus avoiding non

25 specific adorption on microelectrodes and degradation of biological samples through uncontrolled faradic reactions.
Experimental results obtained highlight the possibility for the monitoring at low fixed-frequency of the electric impedance changes provoked by the association of proteic ligands. Here it is 30 illustrated for association reaction between rabbit anti-BSA antibodies in solution and BSA adsorbed onto the PET surface. Thus, it was found that the kinetics of association reaction rate depend on a second order kinetic law with the anti-BSA antibodies concentration. The promising results obtained for the 35 kinetic parameters and the equilibrium association constants between ligands using the present methodology make it a competing method for biosensing. The dielectric PET microchip is also a low cost and flexible material which offers a multiuse chip possibility. It could be used to screen putative partners in 40 biological fluids with regard to a specific protein. Also, the minute volumes of solvent used make it suitable as a detector for a capillary electrophoresis apparatus or to be connected with mass spectrometer.

\section{Acknowledgments}

The authors thank Prof Hubert Girault and Dr Philippe Flückiger for allowing them access to experimental facilities at the EPFL center of MicroNano Technology, Lausanne.

\section{Notes and references}

${ }_{50}{ }^{a}$ CNRS, UMR 8235, Laboratoire Interface et Systèmes Electrochimiques, (LISE),F-75005 Paris, France.*Jean.gamby@upmc.fr

${ }^{b}$ Sorbonne Universités, UPMC Univ Paris 06, UMR 8235, Laboratoire Interfaces et Systèmes Electrochimiques, (LISE), 4 Place Jussieu, F75005, Paris, France.

${ }_{55}{ }^{c}$ Laboratoire d'Electrochimie, Corrosion et de Valorisation Energétique (LECVE), Faculté de Technologie, Université A. MIRA, Béjaia 06000 Algeria.

${ }^{d}$ Sorbonne Universités, UPMC Univ Paris 06, UMR 7622, Laboratoire Biologie des Semences, 4 Place Jussieu, F-75005 Paris, France.

[1] A. Qureshi, Y. Gurbuz, S. Kallempudi and J. H. Niazi, Physical Chemistry Chemical Physics 2010, 12, 9176-9182.

[2] N. J. Ronkainen, H. B. Halsall and W. R. Heineman, Chemical Society ${ }_{65}$ Reviews 2010, 39, 1747-1763.

[3] J. Gamby, M. Lazerges, C. Pernelle, H. Perrot, H. H. Girault and B. Tribollet, Lab on a Chip 2007, 7, 1607-1609.

[4] R. Schirhagl, U. Latif, D. Podlipna, H. Blumenstock and F. L. Dickert, Anal. Chem. (Washington, DC, U. S.) 2012, 84, 3908-3913.

70 [5] R. Davidsson, B. Johansson, V. Passoth, M. Bengtsson, T. Laurell and J. Emneus, Lab on a Chip 2004, 4, 488-494.

[6] F. B. Myers and L. P. Lee, Lab on a Chip 2008, 8, 2015-2031.

[7] J. Homola, Analytical and Bioanalytical Chemistry 2003, 377, 528539.

75 [8] A. J. Tudos and R. B. M. Schasfoort in Chapter 1 Introduction to Surface Plasmon Resonance, Vol. The Royal Society of Chemistry, 2008, pp. 1-14.

[9] A. J. Zemann, E. Schnell, D. Volgger and G. K. Bonn, Analytical Chemistry 1998, 70, 563-567.

80 [10] J. G. A. Brito-Neto, J. A. Fracassi da Silva, L. Blanes and C. L. do Lago, Electroanalysis 2005, 17, 1198-1206. 
[11] J. Liu, J. Wang, Z. Chen, Y. Yu, X. Yang, X. Zhang, Z. Xu and C. Liu, Lab on a Chip 2011, 11, 969-973.

[12] J. Gamby, J.-P. Abid and H. H. Girault, Journal of the American Chemical Society 2005, 127, 13300-13304.

5 [13] J. Gamby, J. P. Abid, M. Abid, J. P. Ansermet and H. H. Girault, Analytical Chemistry 2006, 78, 5289-5295.

[14] G. Schaumburg, Dielectrics Newsletter, Scientific Newsletter for Dielectric Spectroscopy 1997, 08, 5-10.

[15] M. Faure, M. Kechadi, B. Sotta, J. Gamby and B. Tribollet, 10 Electroanalysis 2013, 25, 1151-1158.

[16] J. S. Rossier and H. H. Girault, Lab on a Chip 2001, 1, 153-157.

[17] Z. Wu, H. Jensen, J. Gamby, X. Bai and H. H. Girault, Lab on a Chip 2004, 4, 512-515.

[18] M. Kechadi, J. Gamby, L. Chaal, H. Girault, B. Saidani and B. 15 Tribollet, Electrochimica Acta 2013, 105, 7-14.

[19] M. Kechadi, J. Gamby, L. Chaal, B. Saidani and B. Tribollet, Journal of Flow Chemistry 2013, 3, 81-86.

[20] M. Kechadi, L. Chaal, B. Tribollet and J. Gamby, Analyst 2014, 139, 1492-1497.

20 [21] E. G. G. Spada, P. Giunchedi, World Academy of Science, Engineering and Technology 2011, 52, 245249.

[22] V. Ferraro, R. F. Jorge, I. B. Cruz, P. M. L. Castro and M. E. Pintado, J. Chem. Technol. Biotechnol., 2014, 89, 671-681.

[23] I. Langmuir, Journal of the American Chemical Society 1918, 40, 25 1361-1403.

[24] E. A. James and D. D. Do, Journal of Chromatography 1991, 542, $19-28$.

[25] G. P. Jeppu and T. P. Clement, Journal of Contaminant Hydrology 2012, 129-130, 46-53.

30 [26] A. Kausaite, M. van Dijk, J. Castrop, A. Ramanaviciene, J. P. Baltrus, J. Acaite and A. Ramanavicius, Biochemistry and Molecular Biology Education 2007, 35, 57-63. 